\title{
Curious Associations of Atrial Septal Defect (ASD) with Cyanosis: Elucidating the Missing Links; Retrospective Descriptive Study
}

\author{
Vivek Kumar ${ }^{1 *}$, Gaurav Kumar ${ }^{2}$, Ravi Ramamurthy ${ }^{1}$, Nikhil Tiwari² and Ashutosh Kumar ${ }^{3}$ \\ ${ }^{1}$ Pediatric cardiologist Army Hospital Research and Referral, India \\ ${ }^{2}$ Pediatric cardiothoracic surgeon Army hospital Research and referral, India \\ ${ }^{3}$ Neonatologist Army hospital research and referral, India
}

Submission: February 21, 2019; Published: March 20, 2019

*Corresponding author: Vivek kumar, Army hospital research and referral Dhaula kuan, Delhi 110010, India

\begin{abstract}
Objective: The main objective of this study was to elucidate the missing links in the hemodynamics of varied etiologies associated with ASD, who were referred to our centre with the primary diagnosis of ASD with cyanosis.

Study design: It is a retrospective descriptive study. The patients included were those who were referred to our centre with an echocardiographic diagnosis of ASD with cyanosis. The details of eight patients from Jan 2017 to Dec 2017 were reviewed from the admission registry.

Results: Out of the eight patients, three were Total Anomalous Pulmonary Venous Connection (TAPVC) two endocardial cushion defects, one Idiopathic Pulmonary Hypertension (IPAH), one absent right pulmonary artery and one congenital meth-hemoglobinemia. There were 7 infants (including two neonates) and one 7-year-old child. Male: female ratio was 5:3 (Table 1).

Conclusion: The mechanisms of cyanosis included admixture lesions, abnormal streaming and high RV pressure. These cases emphasized the fact that the ASD in most of the cases was a bystander lesion. The missing links in the hemodynamic puzzle had to be understood to reach at the correct diagnosis.
\end{abstract}

Keywords: Atrial septal defect; Cyanosis; Total anomalous pulmonary venous connection

\section{Introduction}

Ostium secundum ASD commonly shunts from left to right atrium leading to volume overload of right ventricle. However, it can shunt from right to left due to severe pulmonary artery hypertension, and obligatory in admixture lesions like TAPVC. Rarely, it may be so large that there may be a complete mixing of right and left atrial blood. All these scenarios would lead to cyanosis associated with ASD. We describe eight cases in which ASD was associated with central cyanosis albeit with different mechanisms.

Table 1: Demographic, clinical and echo data.

\begin{tabular}{|c|c|c|c|c|}
\hline Patient no & Age & Sex & Presentation diagnosis & Echo diagnosis \\
\hline 01 & 26 days & M & ASD with PPHN & Congenital methemoglobinemia moderate ASD L $>$ R \\
\hline 02 & 15 days & F & ASD with PPHN & Infracardiac TAPVC with arch hypoplasia, moderate PAH small ASD R>L \\
\hline 03 & 2 months & M & Large ASD with PAH & Unobstructed cardiac TAPVC, Moderate PAH with moderate ASD R $>$ L \\
\hline 04 & $4 m o n t h s$ & M & ASD with CHF, cyanosis & Unobstructed supracardiac TAPVC, Severe PAH Recurrent LRTI \\
\hline
\end{tabular}

\section{Study design}

Retrospective analysis of the data was done from Jan 2017 to Dec 2017.

\section{Inclusion criteria}

Patients, who were referred from outside with an echocardiographic diagnosis of ASD with cyanosis were included, and thoroughly evaluated so as to find out the exact etiology of the cyanosis. All these echo-cardiographic diagnosis were done by adult cardiologist. 


\section{Journal of Cardiology \& Cardiovascular Therapy}

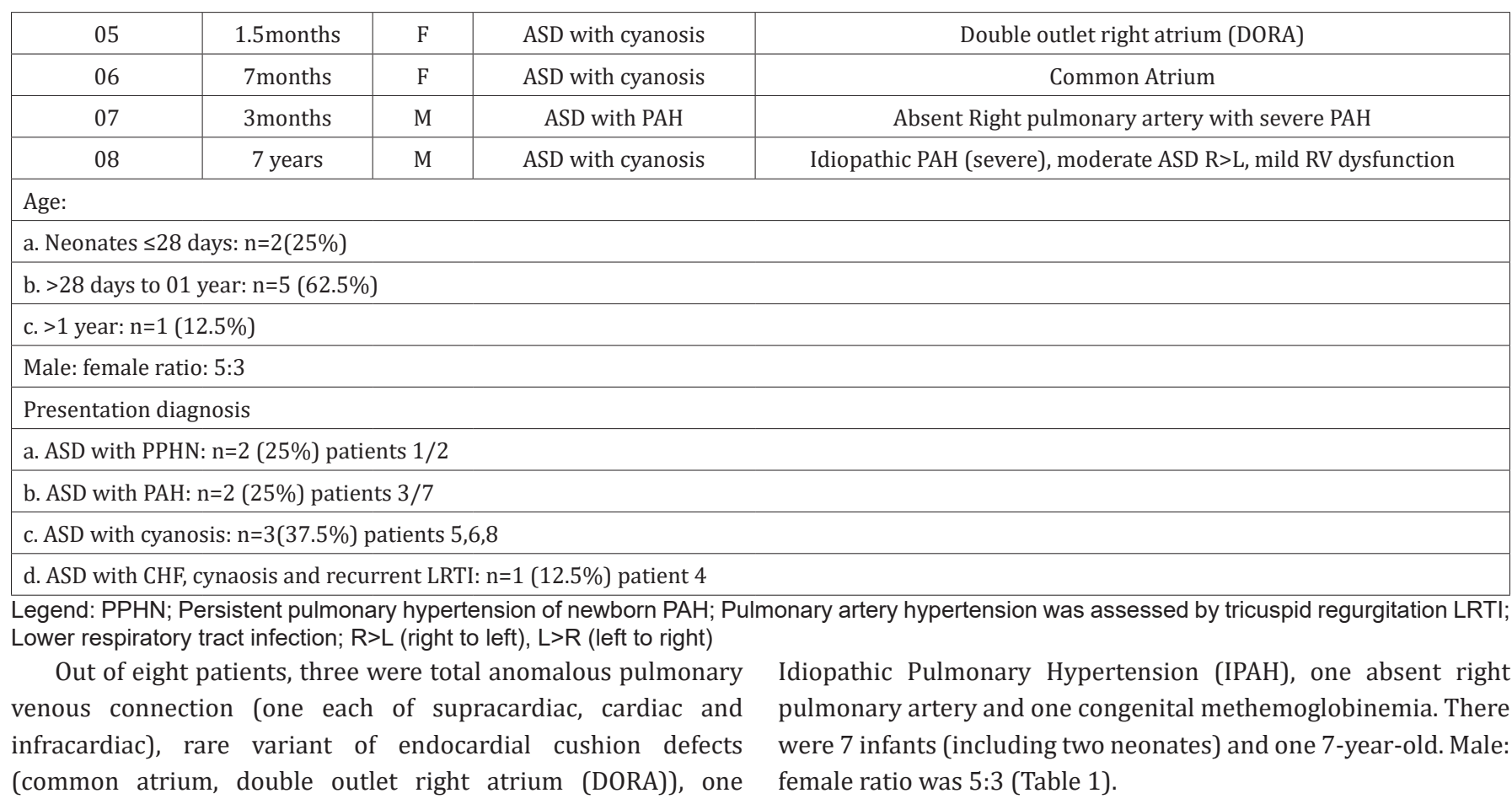

Results

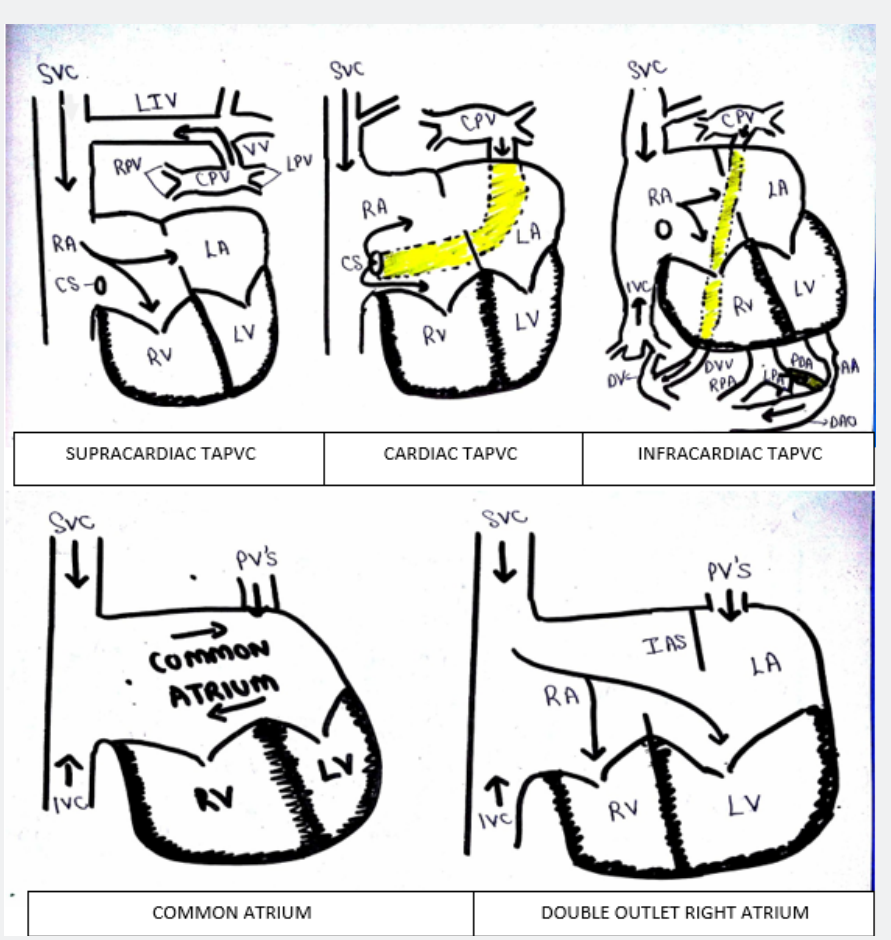

Figure 1: (Hand drawn): Pictorial presentation of hemodynamics in TAPVC and endocardial cushion defects

LEGEND: SVC (superior vena cava), IVC (inferior vena cava), RA (right atrium), LA (left atrium), RV (right ventricle), LV (left ventricle), LIV (left innominate vein), RPV (right pulmonary veins), LPV (left pulmonary veins), CPV (common pulmonary vein formed by the confluence of all four pulmonary veins), VV (vertical vein), CS (coronary sinus), DV (ductus venosus), DVV (Descending vertical vein), AA (aortic arch which is hypoplastic), RPA (right pulmonary artery), LPA (left pulmonary artery), PDA (patent ductus arteriosus which is continuing as descending aorta), DAO (descending aorta), PVs (pulmonary veins), IAS (interatrial septum which is mal aligned to left side)

Patient 1 was saturating $85 \%$ with a diagnosis of persistent pulmonary hypertension of newborn (PPHN). He had failed hyperoxia test (Table 2). Blood was chocolate brown in colour, while Echocardiography (Echo) showed moderate ostium secundum ASD shunting left to right. Meth-hemoglobin levels were $35 \%$ and enzyme NADH cytochrome b5 reductase was 


\section{Journal of Cardiology \& Cardiovascular Therapy}

deficient. Patients 2/3/4 were TAPVCs (admixture lesions) with varied presentation. Cardiac TAPVC (patient 2) saturating 89\% was referred as large ASD left to right with PAH. Patient 3 admitted as a moderate ASD with PPHN, saturating $82 \%$ had infracardiac TAPVC with aortic arch hypoplasia (duct dependent). Final diagnosis was cayler cardiofacial syndrome (triad of absent Depressor anguli oris (DAOM), CHD, 22q11microdeletion+). Third case was an unobstructed supracardiac TAPVC, referral diagnosis being ASD with failure and recurrent respiratory infection. The latter was severely anemic, septic with pneumonia and saturated $82 \%$ in room air (Figure 1 and Table 1 \& 2). Common atrium (patient 6) was another admixture physiology (Figure 1). Patient 5 was double outlet right atrium. These two (patients 5,6) endocardial cushion defects were referred as ASD with cyanosis.
Patient 7 was referred with an echo diagnosis of ASD with PAH. He saturated $82 \%$ in room air increasing to $89 \%$ with oxygen. Echo showed an absent right pulmonary artery. Computerised tomography scan confirmed the diagnosis and also showed massive collateralization of right lung. Patient 8 was an idiopathic PAH patient who was referred as ASD with cyanotic heart disease. He was saturating $80 \%$ in room air.

\section{Conclusion}

ASD normally shunts from left atrium to right due to low right ventricle end-diastolic pressures (RVEdp) leading to volume overload of RV, however if the RVEdp is high as in pressure overload situation of pulmonary hypertension the ASD flow will reverse from right to left atrium leading to cyanosis.

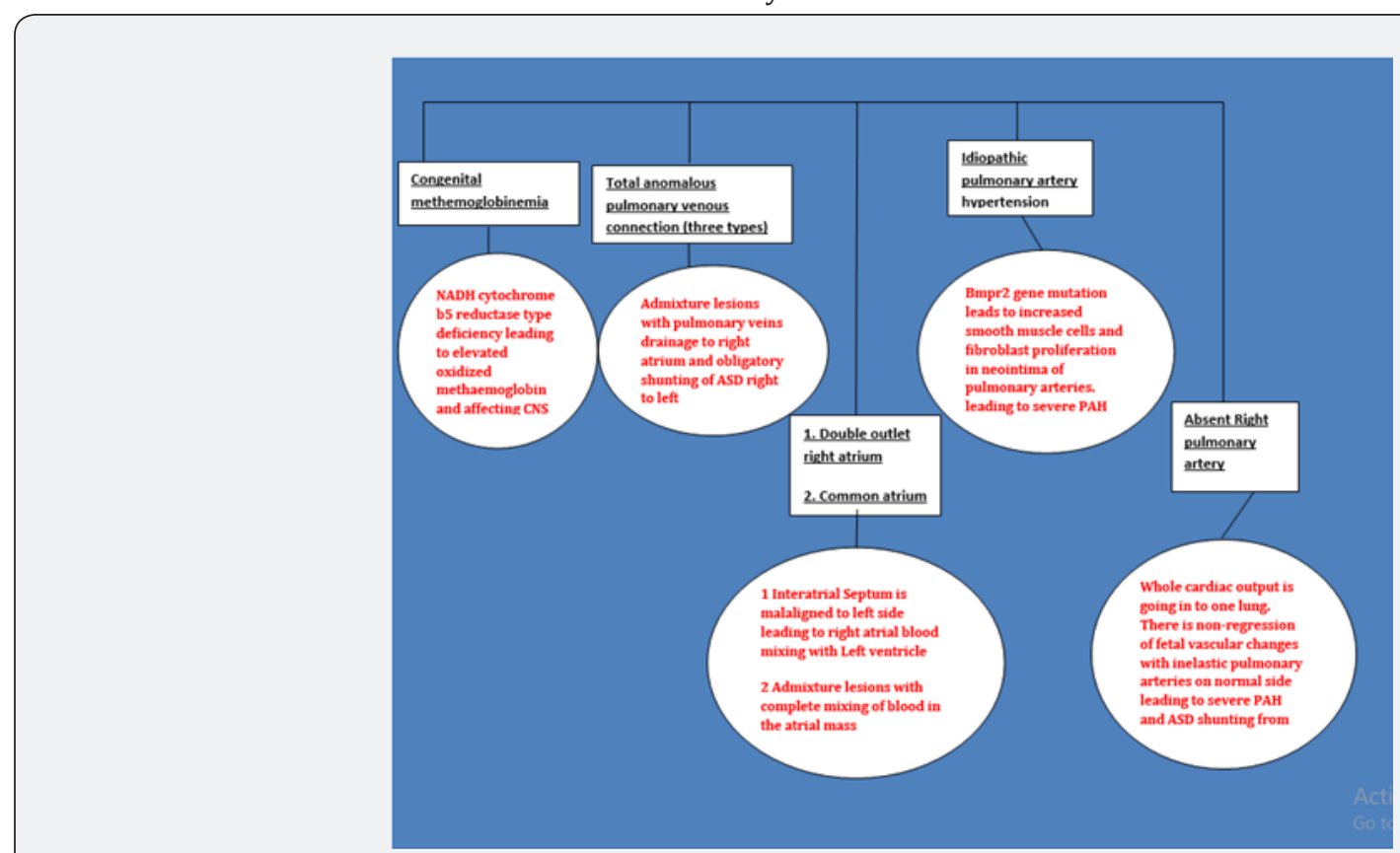

Figure 2: Showing mechanisms of cyanosis.

Table 2: Ancillary investigations, Hemodynamics/pathology and final outcome.

\begin{tabular}{|l|}
\hline Ancillary investigations \\
\hline a. CT scan: $\mathrm{n}=2$ (patients 1,3 ) \\
\hline b. MRI brain: $\mathrm{n}=1$ (patient 2 ) \\
\hline c. Microdeletion 22q11: $\mathrm{n}=1$ (patient 1 ) \\
\hline d. Cytochrome b5 reductase enzyme: $\mathrm{n}=1$ (patient 2) \\
\hline e. Hyperoxia test(failed): $\mathrm{n}=1$ (Patient 1 ) \\
\hline f. Hemogram, Peripheral smear (microcytic hypochromic severe anemia): $\mathrm{n}=1$ (patient 3) \\
\hline Hemodynamics/pathology \\
\hline a. Admixture lesions: $\mathrm{n}=4$ (patients 2,3,4,6) \\
\hline b. Abnormal streaming of deoxygenated blood: $\mathrm{n}=1$ (patient 5 ) \\
\hline c. High RV pressure: $\mathrm{n}=2$ (patients 7,8 ) \\
\hline d. Abnormal hemoglobin: $\mathrm{n}=1$ (patient 1 ) \\
\hline Final outcome \\
\hline a. Operated doing well: $\mathrm{n}=3$ (patients $2,3,6$ ) \\
\hline b. Died secondary to sepsis: $\mathrm{n}=1$ (patient 4 ) \\
\hline c. Medical follow-up: $\mathrm{n}=3$ (patients 1,5,7,8) \\
\hline
\end{tabular}


Congenital methemoglobinemia which was our first case is due to the deficiency of cytochrome b5 reductase deficiency and is a very rare cause of cyanosis in neonates. Methemoglobin (normally<1\%), has limited ability to carry oxygen, results from oxidation of ferrous iron to ferric iron within the haem moiety of hemoglobin. In the absence of enzyme methemoglobin is not converted to active reduced form [1]. The ASD in our case was just a bystander. This patient on follow-up showed global developmental delay and abnormality on neuro-imaging (Table 2).

We had four cases of admixture physiology (Table 2). This is described as a cardiac defect which facilitates complete mixing of the deoxygenated Systemic Venous (SV) blood returning from the tissues and the fully oxygenated pulmonary venous blood from the lungs in a common receiving chamber. It's a physical process so the final saturation depends upon the pulmonary blood flow (Qp) as SV (Qs) blood is relatively constant.

TAPVC has complete admixture of blood in the right atrium (RA) and obligatory shunting of ASD from right to left (Figure 1 \& Figure 2). Hemodynamically if not obstructed TAPVC behaves like an ASD. These lesions have a massive Qp:Qs (3:1 to 4:1) leading to high saturations and mild or visibly no cyanosis [2]. Cardiac TAPVC has hugely dilated coronary sinus on echo, shunting pulmonary venous blood to right atrium, which can be mistaken as an ASD. Supra-cardiac and infra-cardiac TAPVC on echo is missed if proper assessment of pulmonary veins are not done. We had an unusual association of DAOM with infra-cardiac TPAVC, and it should always be remembered that DAOM has almost 50\% association with CHD [3]. Infra-cardiac TAPVC are almost always obstructed and they desaturate, unlike our patient who probably had a favorable steaming of oxygenated IVC blood to LA (akin to fetal circulation). Complete admixture also occurred in common atrium [4] (Figure 1 \& Figure 2).

DORA has an abnormal streaming of deoxygenated blood from RA to Left Ventricle (LV). This happens because there is an abnormal leftward movement of the ventricles and ventricular septum relative to the atria and the atrial septum leading to atrial septum lying well to the left of the ventricular septum. Hence, the RA opens into both the RV and the LV [5] (Figure 1 \& Figure 2).

Patient with absent RPA has whole cardiac output going in to one lung. In such cases it is postulated that there is non regression of fetal vascular changes with inelastic pulmonary arteries on normal side leading to severe PAH and ASD shunting from right to left [6]. Our last patient with idiopathic PAH had pressure overloaded RV and the ASD which was incidentally associated, shunted right to left to produce cyanosis.

These constellation of cases show that the ASD in most of them was just a part of the complex mechanism leading to cyanosis. On presentation the puzzle of hemodynamics was incomplete in these patients. We could have probably picked up few cases in perinatal period if standard neonatal pulse oximetry screening would have been done. The final outcome of admixture lesions is gratifying, however we lost one supra-cardiac TAPVC due to sepsis. Patients with Idiopathic PAH, absent RPA (presenting in infancy) and congenital methemoglobinemia does not have good long-term prognosis (Table 2).

\section{References}

1. Yassin MA, Soliman DS (2017) Congenital Methemoglobinemia Under Diagnosed Condition with Variable Therapeutic Options. Blood 130: 4746.

2. Gupta CD, Satpathy M (2008) Total anomalous pulmonary venous connections. In: Sathpathy M, Mishra BR (eds.), Clinical Recognition of Congenital Heart Diseases. New Delhi: Jaypee Brothers Medical Publishers p. 317-324.

3. Lin DS, Huang FY, Lin SP, Chen MR, Kao HA, et al. (1997) Frequency of associated anomalies in congenital hypoplasia of depressor angularis oris muscle: a study of 50 patients. Am J Med Genet 71(2): 215-218.

4. Sajeev CG, Roy TNS, Venugopal K (2002) Common atrium in a child with Ellis-Van Creveld syndrome. Heart 88(2): 142.

5. Brancaccio G, Amodeo A, Rinelli G, Filippelli S, Sanders SP, et al. (2007) Double-Outlet right atrium: anatomic and clinical considerations. Ann Thorac Surg 83(2): 619-621.

6. Haworth SG, de Leval M, Macartney FJ (1981) Hypoperfusion and hyperperfusion in the immature lung. Pulmonary arterial development following ligation of the left pulmonary artery in the newborn pig. J Thorac Cardiovasc Surg 82(2): 281-292.

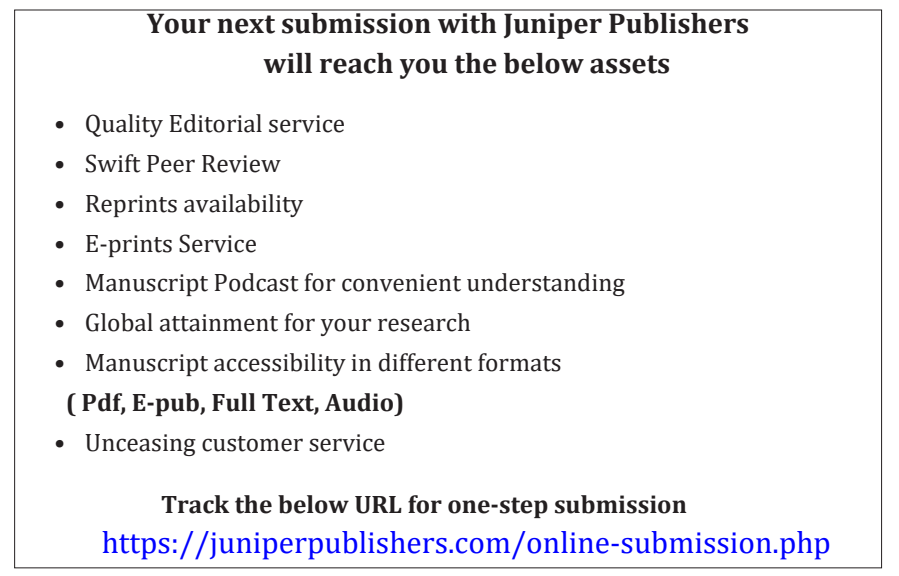

